\title{
Dinâmica vegetacional em pastagem natural submetida a diferentes sistemas de manejo
}

\author{
Vegetation dynamics of natural grassland under different management systems
}

\author{
Marcos da Silva Brum ${ }^{\mathrm{I}}$ Fernando Luiz Ferreira de Quadros ${ }^{\text {II }}$ Jorge Dubal Martins ${ }^{\text {III }}$ \\ Duilio Guerra Bandinelli ${ }^{\mathrm{I}}$ Guilherme Ebling Rossi ${ }^{\mathrm{IV}}$ Everton Daniel $^{\mathrm{IV}}$ \\ Adriano Rudi Maixner ${ }^{\mathrm{I}}$ Alexandre Coradini Fontoura da Silva ${ }^{\mathrm{I}}$ \\ Naíme de Barcellos Trevisan ${ }^{\mathrm{I}}$ \\ Niumar Dutra Auréliov
}

\section{RESUMO}

A dinâmica da composição florística de uma pastagem natural foi avaliada para estimar os efeitos de sistemas de pastejo e introdução de espécies hibernais. $O$ experimento foi realizado em São Gabriel, na região da Depressão Central, Rio Grande do Sul. O período de pastejo foi entre 01/11/2004 e 23/12/2004, totalizando 53 dias. Foram realizados dois levantamentos da composição florística, sendo o primeiro antes do início do pastejo (23/09/2004) e o segundo após o término do período experimental (27/01/2005). Os tratamentos testados foram: pastagem natural melhorada com introdução de espécies hibernais sob pastejo rotativo (CNM); pastagem natural sob pastejo contínuo (CNC) e pastagem natural sob pastejo rotativo (CNR), sendo distribuídos em delineamento inteiramente casualizado, com duas repetições. Os animais utilizados no pastejo foram ovelhas com cordeiros da raça Corriedale. Os dados foram submetidos à análise multivariada baseada em ordenação e testes de aleatorização. No primeiro levantamento botânico, o tratamento CNM diferiu $(P=0,0001)$ do CNC e CNR pela elevada participação das espécies introduzidas, enquanto CNC e do CNR foram semelhantes $(P=0,6742)$ na composição de espécies. Os tratamentos CNM e CNC foram diferentes $(P=0,0017)$ no segundo levantamento. O efeito do pastejo rotativo, a introdução de espécies e a adubação são determinantes para mudanças na composição da vegetação. A vegetação sob efeito de pastejo rotativo apresentou trajetórias que convergem para uma situação de semelhança na composição de espécies.

Palavras-chave: composição florística, melhoramento, métodos de pastejo, ovinos.

\section{ABSTRACT}

Floristic composition's dynamics of a natural grassland was evaluated to estimate the effects of grazing systems and introduction of cool season species. The experiment was held in São Gabriel, in the region of Depressão Central, Rio Grande do Sul, Brazil. The grazing period was from 01/11/ 2004 to 23/12/2004, totaling 53 days. Floristic composition were evaluated before the beginning of the grazing period (23/ 09/2004) and after its end (27/01/2005). Tested treatments were: natural grassland improvement with introduction of cool season species under rotative grazing (CNM); natural grassland under continuous grazing (CNC) and natural grassland under rotative grazing (CNR), distributed in an entirely randomized design, with two replicates. Corriedale breed ewes, with their lambs, were tester grazing animals. Data were submitted to multivariate analysis based in ordination and randomization testing. In the first survey, the treatment CNM differed $(P=0.0001)$ from $C N C$ and CNR due to the high contribution of the introduced species, while CNC and CNR were similar $(P=0.6742)$ in species' composition. Treatments CNM and CNC were different $(P=0.0017)$ in the second evaluation. The effect of rotative grazing, introduction of species and fertilization seems to be decisive for changes in floristic composition. Vegetation under rotative grazing presented trajectories that converge to a situation of similarity in the species' composition.

Key words: floristic composition, grazing methods, improvement, sheep.

\footnotetext{
IPrograma de Pós-graduação em Zootecnia (PPGZ), Universidade Federal de Santa Maria (UFSM), Campus Camobi, 97105-900,

Santa Maria, RS, Brasil. E-mail: marcossbrum@yahoo.com.br. Autor para correspondência.

IIDepartamento de Zootecnia, UFSM, Santa Maria, RS, Brasil. E-mail: flfquadros@yahoo.com.br.

IIIFundação Estadual de Pesquisa Agropecuária (FEPAGRO), São Gabriel, RS, Brasil.

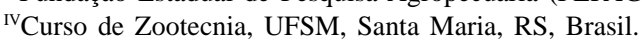

${ }^{\mathrm{v}}$ Curso de Agronomia, UFSM, Santa Maria, RS, Brasil.
} 


\section{INTRODUÇÃO}

Na região sudoeste do Rio Grande do Sul, existe um grande número de produtores que vivem basicamente da pecuária de corte (bovinos e ovinos). Nesta região, ocorrem os chamados campos mistos, com uma vegetação típica de transição entre os campos grossos do Planalto e os campos finos da Campanha, onde a vegetação campestre é composta por plantas de porte cespitoso, herbáceo e arbustivo, com muitas espécies de compostas e de andropogôneas. Entre as espécies que compõem o estrato inferior, Paspalum notatum é a que domina no topo das coxilhas e, à medida que a umidade aumenta, há maior freqüência de Axonopus affinis e Paspalum pumilum (BOLDRINI, 1997).

O conhecimento da dinâmica da vegetação e sua resposta às alterações no manejo e aplicação de insumos são importantes para estudar como determinada prática modifica a freqüência de espécies importantes, tanto do ponto de vista da produção animal, quanto da conservação do ecossistema. Portanto, faz-se necessário conhecer melhor os fatores que afetam a sucessão vegetal num determinado ambiente (NABINGER, 2000).

Em pastagens, o pastejo é a forma mais comum de mudança espacial e temporal na estrutura e dinâmica de comunidades, tendo como principal efeito a perturbação provocada pelo pisoteio ou pela remoção do material verde que abre espaços na comunidade vegetal, permitindo a colonização e o estabelecimento de diferentes espécies (PANDEY \& SINGH, 1991).

O melhoramento da pastagem natural também implica modificações na flora da pastagem, seja em função do aporte de nutrientes via adubação, da introdução de espécies exóticas ou mesmo da associação destes fatores (COELHO FILHO \& QUADROS, 1995). O melhoramento através da introdução de espécies cultivadas (gramíneas e leguminosas de estação fria) é uma prática recomendada para enfrentar a estacionalidade produtiva das pastagens naturais. A introdução de espécies hibernais melhora a qualidade da pastagem, incrementando a produção de massa seca durante a estação fria e também a produção das espécies nativas durante a primaveraverão (RIZO et al., 2004). Esta prática de melhoramento também é importante por envolver baixos custos, manter a estrutura física do solo e não eliminar as espécies nativas, que, em determinadas condições, podem contribuir para melhorar a composição de forragem.

Com relação a sistemas de pastejo, PERIN (1990) conduziu um experimento em pastagem natural melhorada sob pastejo contínuo ou rotativo e não observou diferenças entre os sistemas de pastejo, tanto na produção de matéria seca, quanto na produção animal. Já BARBOSA et al. (2004), avaliando diferentes intensidades e métodos de pastejo em pastagem de azevém anual (Lolium multiflorum Lam.) pastejado com cordeiros, verificou superioridade do pastejo contínuo e da intensidade de pastejo baixa no desempenho individual dos animais. As cargas animais utilizadas no pastejo rotativo foram superiores às utilizadas no pastejo contínuo. Com relação aos ganhos de peso por unidade de área, nenhuma diferença foi verificada entre os tratamentos. Portanto, afirmaram que a quantidade de forragem em oferta para o animal é a principal determinante da produtividade do sistema, mais do que os métodos de pastejo.

NABINGER (1998) comentou que, no sistema de pastejo rotativo, é possível manter um equilíbrio estável entre consumo de forragem e crescimento da pastagem e assim evitar um excesso de acúmulo de material senescente e o desenvolvimento de áreas de rejeição com alto conteúdo de material morto. Considerando os efeitos do pastejo contínuo, esse mesmo autor afirma que, numa condição de alta oferta de forragem, o pastejo contínuo pode ser mais favorável que o pastejo rotativo, pois mantém um Índice de Área Foliar (IAF) constante ao longo da estação favorável. Contudo, isto, inevitavelmente, também contribui para uma alta taxa de senescência. Portanto, existem divergências sobre as vantagens e desvantagens de cada sistema.

Sobre os efeitos de sistemas pastejo na composição botânica, MILLOT (1991) afirmou que, em sistema de pastejo contínuo, não se conta com períodos de repouso da pastagem, e esse manejo favorece as espécies prostradas (Paspalum notatum, Axonoppus spp.), cujos rebrotes não dependem de substâncias de reserva, mas sim de área foliar remanescente, sendo mais sensíveis à competição por luz do que as espécies eretas. Ao contrário, períodos de descanso vão favorecer espécies com hábito de crescimento ereto, que, na estação de crescimento, competem em vantagem com as espécies citadas anteriormente.

Diante da escassez de informações quantitativas para as condições das pastagens naturais do Sul do Brasil, o presente trabalho procura estabelecer relações entre os sistemas de manejo e a estrutura da vegetação campestre. O objetivo deste trabalho foi avaliar efeitos de sistemas de pastejo e introdução de espécies hibernais sobre a dinâmica da composição florística de uma pastagem natural em São Gabriel, RS. 


\section{MATERIAL E MÉTODOS}

O experimento foi conduzido no período de 01/11 a 23/12/2004, em área pertencente ao Centro de Pesquisas de Forrageiras, da Fundação Estadual de Pesquisa Agropecuária (FEPAGRO), em São Gabriel, RS, situado na região fisiográfica da Depressão Central, entre as coordenadas $30^{\circ} 20^{\prime} 27^{\prime \prime}$ S e $54^{\circ} 19^{\prime} 01^{\prime \prime} \mathrm{W}$. A altitude média é de $109 \mathrm{~m}$ acima do nível do mar e o clima da região é subtropical úmido (Cfa), com verões muito quentes segundo a classificação de Köppen. O solo da área experimental pertence à unidade de mapeamento Alto das Canas, sendo um argissolo vermelho distrófico latossólico (EMBRAPA, 1999).

Os tratamentos testados foram: pastagem natural melhorada com introdução de espécies hibernais sob pastejo rotativo (CNM); pastagem natural sob pastejo contínuo (CNC) e pastagem natural sob pastejo rotativo (CNR), arranjados em delineamento experimental inteiramente casualisado com duas repetições. Foram utilizadas áreas de oito hectares para o CNC, dividido em dois potreiros, sendo que cada potreiro constituía uma repetição; de 4,8ha para o CNM, divididos em 20 potreiros, com área média de 0,24ha, sendo que cada 10 potreiros constituíam uma repetição; e 7,3 ha para o CNR, divididos em 20 potreiros com área média de 0,36 ha, sendo que cada 10 potreiros constituiam uma repetição. A divisão dos potreiros foi realizada por meio de cerca elétrica.

Na área do tratamento CNM, foi feita a correção da acidez do solo no início do mês de maio, sendo aplicadas $3 t$ ha $^{-1}$ de calcário, elevando-se a saturação de bases para 65\%. A introdução das espécies hibernais ocorreu no dia 18/05/2004, por meio de semeadura direta, utilizando-se $38 \mathrm{~kg} \mathrm{ha}^{-1}$ de sementes de azevém anual (Lolium multiflorum Lam.) cv. "Comum”, semeadas em linhas, com uma densidade de $5 \mathrm{~kg} \mathrm{ha}^{-1}$ de trevo vermelho (Trifolium pratense L.) cv. "E-116” e de $8 \mathrm{~kg} \mathrm{ha}^{-1}$ de cornichão (Lotus corniculatus L.) cv. “São Gabriel”, semeadas a lanço. Não foi utilizado herbicida para controle da vegetação natural, sendo que, no dia da semeadura, a pastagem natural se apresentava rapada por utilização de pastoreio intenso com bovinos da estação experimental. A adubação de base ocorreu por ocasião da semeadura, utilizando-se $350 \mathrm{~kg} \mathrm{ha}^{-1}$ da fórmula $02-$ 25-25(NPK).

Foram utilizadas de 27 a 33 ovelhas-teste, com seus respectivos cordeiros (machos e fêmeas), por tratamento, e um número variável de reguladores. O número de animais-teste variou em função da perda por mortalidade ou do furto de animais durante o período experimental. Os animais utilizados eram da raça Corriedale, com idades e pesos médios de 36 meses e 40,2kg, 3 meses e 22,8kg, para ovelhas e cordeiros, respectivamente. O início do pastejo foi em 01 de novembro de 2004, devido à instalação das cercas divisórias não ter sido concluída anteriormente. $\mathrm{O}$ período experimental foi até 23 de dezembro, totalizando 53 dias, quando os cordeiros foram desmamados. Foi utilizada a carga animal média de 561, 461 e 319kg de PV ha $^{-1}$ nos tratamentos CNM, CNR e CNC, respectivamente. Este período foi caracterizado por uma forte estiagem.

Para o CNC, o método de pastejo foi contínuo, com taxa de lotação variável, utilizando a técnica de animais reguladores (MOTT \& LUCAS, 1952), sendo que a carga animal foi ajustada quando necessário, com o objetivo de manter uma oferta de $10 \%$ (10kg de MS $100 \mathrm{~kg}_{\text {de }} \mathrm{PV}^{-1}$ ). Já nos tratamentos CNM e CNR, o método de pastejo foi rotativo, e os períodos de ocupação dos potreiros foram variáveis em número de dias, com o objetivo de atingir uma massa de forragem residual de $1.000 \mathrm{Kg}$ de $\mathrm{MS} \mathrm{ha}^{-1}$. No tratamento CNR, o período de utilização e descanso dos potreiros foi em média de 5 e 47 dias, respectivamente, e, no tratamento CNM, o período de utilização e descanso dos potreiros foi em média de 4 e 38 dias, respectivamente.

Foram realizados dois levantamentos da composição florística, sendo o primeiro antes do início do pastejo (23 de setembro de 2004) e o segundo após o término do período experimental (27 de janeiro de 2005).

Em cada repetição, foram demarcadas três transectas de aproximadamente $30 \mathrm{~m}$, localizadas de forma a apresentar emcondições de vegetação e relevo semelhantes aos da área total de cada repetição. No tratamento CNC, as três transectas foram demarcadas sobre um mesmo potreiro, que representava uma repetição. Nos tratamentos CNM e CNR, cada transecta foi demarcada em um potreiro, sendo selecionados três potreiros dentre os que representavam uma repetição. Em cada transecta, foram avaliados 10 quadros. Em cada quadro de $0,25 \mathrm{~m}^{2}$ de área, foi descrita a composição florística, através da listagem das espécies componentes e respectivas quantidades de biomassa obtidas por estimativa visual. A avaliação da disponibilidade total de matéria seca, da participação relativa das principais espécies na matéria seca e da freqüência relativa das demais espécies da vegetação nativa foi realizada, através de dupla amostragem, seguindo os procedimentos de campo do Software Botanal (TOTHILL et al., 1992), usando planilha de cálculo desenvolvida por MARTINS \& QUADROS (2004). 
Os dados foram submetidos à análise multivariada baseada em ordenação e testes de aleatorização, utilizando o Software Multiv (PILLAR, 1997). Para balancear as diferenças de magnitude das participações de diferentes espécies na biomassa total, especialmente no segundo levantamento, foi usada a transformação de padronização pelos totais marginais, em que cada observação é dividida pelo total da variável. Do total de espécies identificadas, foram selecionadas para análise as espécies que apareciam, no mínimo, em três transectas avaliadas por levantamento.

\section{RESULTADOS E DISCUSSÃO}

A análise foi realizada com a composição florística média de cada transecção (média de 10 quadros avaliados por transecção). Nos dois levantamentos realizados, foram identificadas 77 espécies. Na figura 1, são apresentadas as trajetórias da composição botânica da pastagem, no espaço de ordenação, quando submetida aos tratamentos. Foi verificada diferença significativa entre períodos $(\mathrm{P}=0,0001)$ e interação tratamento x período $(\mathrm{P}=0,0001)$. Pode-se observar que a composição botânica no tratamento CNC não se alterou do primeiro para o segundo levantamento. No tratamento CNR, mesmo com um período relativamente curto de avaliação, a composição botânica foi influenciada pelo sistema de manejo, passando de uma condição inicial semelhante ao CNC, onde havia uma maior contribuição de Paspalum notatum na matéria seca (MS) total, para uma condição similar ao tratamento CNM, com maior contribuição de espécies como Andropogon lateralis, Erianthus angustifolius e Eryngium horridum (Tabela 1). Porém, o tratamento CNR teve uma trajetória mais curta do que a do tratamento CNM, indicando uma menor variação na composição de espécies ao longo do período experimental. Entre os fatores que afetam a composição da vegetação, a fertilidade do solo exerce uma influência mais marcante que o manejo do pastejo (DURU et al., 2005).

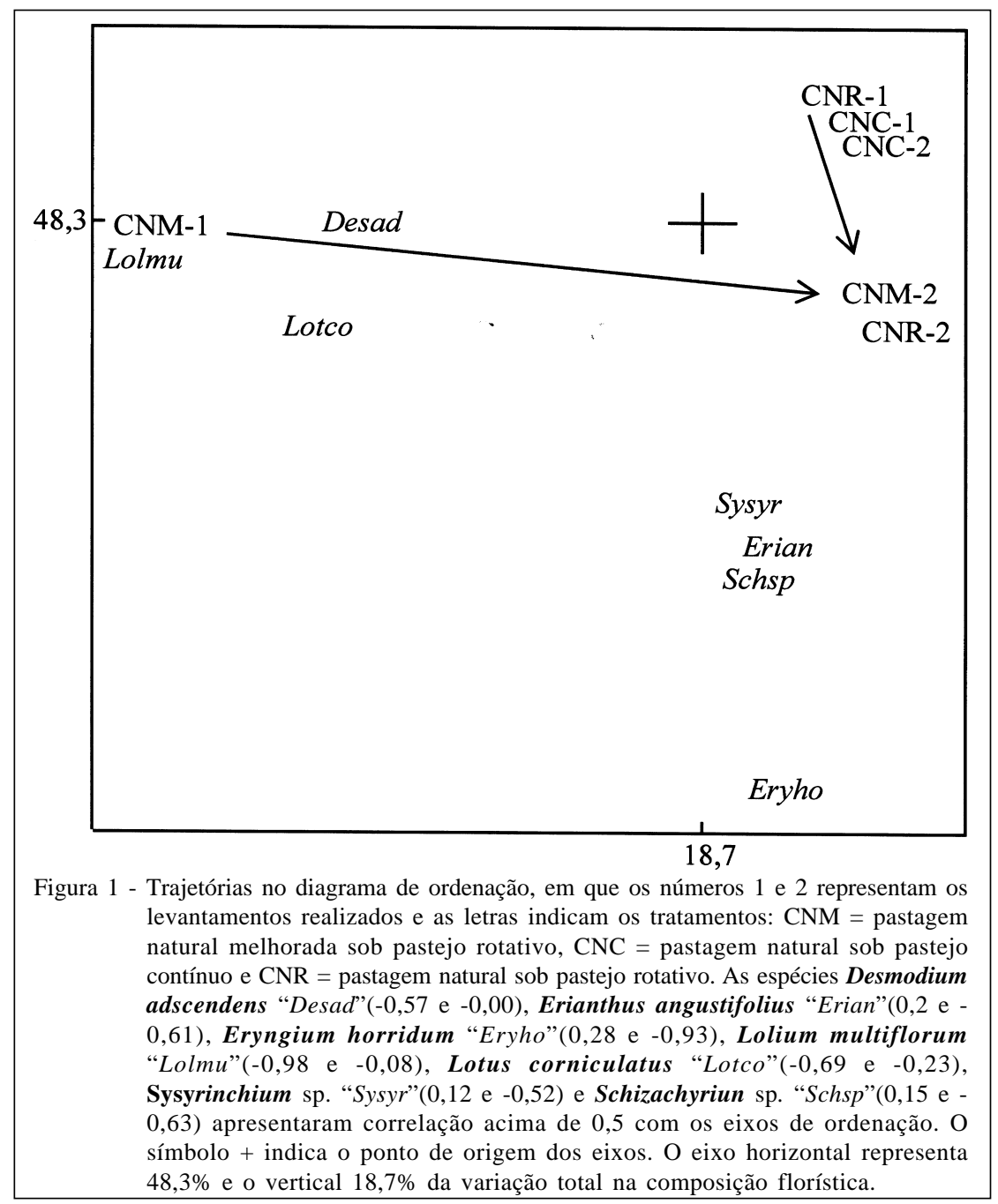

Ciência Rural, v.37, n.3, mai-jun, 2007. 
Tabela 1 - Contribuição média em (kg.ha-1 de MS) das espécies com maior correlação com os eixos I e II do plano de ordenação, nos dois levantamentos botânicos.

\begin{tabular}{|c|c|c|c|}
\hline \multirow{2}{*}{ Espécie } & \multicolumn{3}{|c|}{ Levantamento 1 (23/09/2004) } \\
\hline & CNM & CNC & CNR \\
\hline Lolium multiflorum & $2.396,8 \mathrm{a} *$ & $0 \mathrm{~b}$ & $0 \mathrm{~b}$ \\
\hline Lotus corniculatus & 29,5 a & $0 \mathrm{~b}$ & $0 \mathrm{~b}$ \\
\hline Paspalum urvillei & 1,3 a & 0,3 a & $0 \mathrm{a}$ \\
\hline Sida rhombifolia & 3,8 a & $0 \mathrm{~b}$ & $1,2 \mathrm{a}$ \\
\hline Paspalum notatum & $80,8 \mathrm{~b}$ & $342,0 \mathrm{a}$ & 381,0 a \\
\hline Desmodium adscendens & 3,1 a & 1,9 a & $0,1 \mathrm{a}$ \\
\hline Trifolium pratense & 426,2 a & $0 \mathrm{~b}$ & $0 \mathrm{~b}$ \\
\hline Eryngium horridum & 293,7 a & 318,7 a & 198,1 a \\
\hline Taraxacum officinale & $2,8 \mathrm{a}$ & 2,6 a & $3,2 \mathrm{a}$ \\
\hline \multirow{2}{*}{ Espécie } & \multicolumn{3}{|c|}{ Levantamento $2(27 / 01 / 2005)$} \\
\hline & CNM & CNC & CNR \\
\hline Andropogon lateralis & 11,6 a & 558,8 b & $759,4 \mathrm{~b}$ \\
\hline Eragrostis plana & 74,4 a & 88,2 a & 222,8 a \\
\hline Borreria verticillata & 0 a & 1,6 a & $3 \mathrm{a}$ \\
\hline Relbunium richardianum & $1,1 \mathrm{a}$ & 3,8 a & 8 a \\
\hline Dichondra sericea & 5,9 a & 3,8 a & $11,3 \mathrm{a}$ \\
\hline Richardia stellaris & $0 \mathrm{a}$ & 2,4 a & $2,9 \mathrm{a}$ \\
\hline Stylosanthes montevidensis & $0,8 \mathrm{a}$ & $0,3 \mathrm{a}$ & $1,6 \mathrm{a}$ \\
\hline Pfaffia tuberosa & $0 \mathrm{a}$ & $0 \mathrm{a}$ & $2,7 \mathrm{a}$ \\
\hline Eryngium horridum & $1.027,4 \mathrm{a}$ & 458 a & 737,8 a \\
\hline Panicum milioides & $2,1 \mathrm{a}$ & $3,4 \mathrm{a}$ & $1,8 \mathrm{a}$ \\
\hline Orthopappus angustifolius & $4 \mathrm{a}$ & $3 \mathrm{a}$ & 4,4 a \\
\hline Aspilia montevidensis & $0,4 \mathrm{a}$ & $4,2 \mathrm{a}$ & $3,2 \mathrm{a}$ \\
\hline Schizachyrium spicatulum & $2,7 \mathrm{a}$ & $0,7 \mathrm{a}$ & $4,1 \mathrm{a}$ \\
\hline Erianthus angustifolius & 255,2 a & 110,2 a & 293,9 a \\
\hline Coelorhachis selloana & 3 a & 1,8 a & $4,2 \mathrm{a}$ \\
\hline
\end{tabular}

*Letras minúsculas distintas na mesma linha diferem entre si $(\mathrm{P}=0,0614)$.

No primeiro levantamento, pode-se observar que existe uma semelhança entre os tratamentos CNC e CNR ( $\mathrm{P}=0,6742)$, enquanto o tratamento CNM diferiu estatisticamente $(\mathrm{P}=0,0001)$ tanto do $\mathrm{CNC}$, como do CNR. Esta diferença se deve principalmente à elevada participação das espécies introduzidas (azevém, trevo vermelho e cornichão) no tratamento CNM (Tabela 1). Esta elevada participação das espécies introduzidas faz com que haja uma diminuição da contribuição das espécies nativas devido à competição que as espécies introduzidas exercem sobre as espécies nativas nesta época do ano. Efeitos semelhantes desta competição foram observados por BANDINELLI et al. (2005) em pastagem natural com introdução de azevém e trevo vesiculoso. A similaridade entre os tratamentos CNC e CNR pode ser atribuída aos manejos semelhantes que as duas áreas vinham recebendo antes do início do experimento. Nestes tratamentos, pode-se observar que Paspalum notatum foi a espécie com maior contribuição na composição botânica, com elevada correlação nos dois tratamentos, sendo observada uma contribuição média de 342 e $381 \mathrm{~kg} \mathrm{ha}^{-1}$ de MS nos tratamentos CNC e CNR, respectivamente, não diferindo entre esses tratamentos $(\mathrm{P}=0,6934)$ e diferindo $(\mathrm{P}=0,0147)$ do tratamento CNM, onde sua contribuição foi de $80,8 \mathrm{~kg}$ ha $^{-1}$ de MS (Tabela 1).

No segundo levantamento botânico, foi observada diferença entre os tratamentos CNM e CNC $(\mathrm{P}=0,0017)$, não sendo observada a diferença entre CNM e CNR $(\mathrm{P}=0,3859)$ e entre CNC e CNR $(\mathrm{P}=0,6865)$. A diferença na composição de espécies entre os tratamentos CNM e CNC pode ter sido em função da adubação recebida pelo tratamento CNM, que favoreceu espécies com estratégias de captura dos recursos do ambiente, como luz e nutrientes (DURU et al., 2005; LAVOREL \& GARNIER, 2002), do que propriamente pelos sistemas de pastejo utilizados. Esta hipótese pode ser reforçada pela ausência de diferença 
significativa entre CNC e CNR no segundo levantamento. Esta ausência de diferença também pode ser justificada pelo curto período de pastejo a que foram submetidos os tratamentos. Entretanto, pode ser observada na figura 1 uma tendência de mudança na composição botânica da pastagem natural quando submetida a diferentes sistemas de pastejo. No primeiro levantamento, os tratamentos CNC e CNR apareciam em posições muito semelhantes no espaço de ordenação e, no segundo levantamento, ocupavam lugares diferentes neste espaço. $\mathrm{O}$ fato de que o CNM e o CNR foram semelhantes no segundo levantamento e submetidos ao pastejo rotativo demonstra que alternativas de manejo podem ser tão importantes quanto a introdução de espécies e a adubação, visando a favorecer determinadas espécies. No tratamento CNM as espécies introduzidas não apresentaram contribuição no segundo levantamento (Tabela 1 ), o que pode ser explicado pelo fato de que espécies anuais como Lolium mutiflorum tendem a apresentar alta contribuição apenas durante a sua estação de crescimento. Neste tratamento, houve um rápido retorno da contribuição de espécies nativas na vegetação, justificado pelo fato de não ter sido usado nenhum herbicida na dessecação do campo nativo para a introdução das espécies cultivadas de estação fria. Pode-se observar também a elevada contribuição da espécie nativa Andropogon lateralis, que apresentou a correlação mais alta com os eixos de ordenação no segundo levantamento, possuindo alta contribuição na MS nos tratamentos CNC e CNR (Tabela 1). O tratamento CNC tende a apresentar maior diversidade de espécies. MILCHUNAS et al. (1988) salientam que a maior riqueza florística é encontrada em áreas sob pastejo intenso. Esta tendência pode ser justificada pelo fato de que, nos tratamentos sob pastoreio rotativo, a composição botânica tende para a dominância de espécies cespitosas como Erianthus angustifolius ou Andropogon Iateralis (Tabela 1). Os períodos de descanso fazem com que, na estação de crescimento, estas espécies possam competir em vantagem com as espécies de hábito de crescimento prostrado. A espécie Eryngium horridum, que, nesta época, encontra-se em estádio reprodutivo, também apresentou alta contribuição na MS nos tratamentos sob pastejo rotativo (Tabela 1), principalmente no CNM. Esta pode ter sido favorecida pela adubação utilizada, pelos períodos de descanso e pela rejeição dos ovinos em pastejá-la, o que proporcionou grande acúmulo de MS neste período. Já no CNC, esta espécie teve participação semelhante na MS nos dois levantamentos realizados (Tabela 1).

\section{CONCLUSÕES}

O efeito do pastejo rotativo, a introdução de espécies e a adubação são determinantes nas mudanças na composição da vegetação da pastagem natural, favorecendo espécies cespitosas. As trajetórias da vegetação sob efeito de pastejo rotativo convergem para uma situação de semelhança na composição de espécies, independentemente da introdução de novas espécies.

\section{REFERÊNCIAS}

BANDINELLI, D.G. et al. Composição florística de pastagem natural afetada por fontes de fósforo, calagem e introdução de espécies forrageiras de estação fria. Ciência Rural, Santa Maria, v.35, n.1, p.84-91, 2005.

BARBOSA, C.M.P. et al. Produção de cordeiros em pastagem de azevém anual ("Lolium multiflorum” Lam) manejada em diferentes intensidades e métodos de pastejo. In: REUNIÃO ANUAL DA SOCIEDADE BRASILEIRA DE ZOOTECNIA, 41., 2004, Campo Grande, MS. Anais... Campo Grande: Sociedade Brasileira de Zootecnia, 2004. 1CD.

BOLDRINI, I.I. Campos do Rio Grande do Sul: caracterização fisionômica e problemática ocupacional. Porto Alegre: Instituto de Biociências, 1997. 39p. (Boletim do Instituto de Biociências, 56).

COELHO FILHO, R.C.; QUADROS, F.L.F de. Produção animal em misturas forrageiras de estação fria semeadas em uma pastagem natural. Ciência Rural, Santa Maria, v.25, n.2, p.289-293, 1995.

DURU M. et al. Functional diversity in low-input grassland farming systems: characterisation, effect and management. Agronomy Research, Saku, v.3, n.2, p.125-138, 2005.

EMBRAPA. Sistema brasileiro de classificação de solo. Brasília: EMBRAPA, 1999. 412p.

LAVOREL S.; GARNIER E. Predicting changes in community composition and ecosystem functioning from plant traits: revisiting the Holy Grail. Functional Ecology v.16, n.5, p.545556, 2002.

MARTINS, C.E.N.; QUADROS, F.L.F. de. BOTANAL: desenvolvimento de uma planilha eletrônica para avaliação de disponibilidade de matéria seca e composição florística de pastagens. In: REUNIÓN DEL GRUPO TÉCNICO REGIONAL DEL CONO SUR EN MEJORAMIENTO Y UTILIZACIÓN DE LOS RECURSOS FORRAJEROS DEL ÁREA TROPICAL Y SUBTROPICAL - GRUPO CAMPOS, 2004, Salto, Uruguai. Memorias... Salto: FAO-Grupo Campos, 2004. v.1, p.229231.

MILCHUNAS, D.G. et al. A generalized model of the effects of grazing by large herbivores on grassland community structure. American Naturalist, Chicago, v.132, n.1, p.87-106, 1988. 
MILLOT, J.C. Manejo del pastoreo y su incidencia sobre la composicion botanica y produtividad del campo natural: Pasturas y produccion animal em áreas de ganaderia extensiva. Montevideo, Uruguai: INIA, 1991. 266p. (Série técnica, 13).

MOTT, G.O.; LUCAS, H.L. The desing conduct and interpretation of grazing trials in cultivated and improved pastures, Pensylvania. In: INTERNATIONAL GRASSLAND CONGRESS, 6., 1952, Pensylvania. Proceedings... Pensylvania: State College, State College, 1952. v.6, p.13801395.

NABINGER, C. Manejo e melhoramento de campo nativo. In: SEMINÁRIO PASTOS, PASTAGENS E SUPLEMENTOS 8., 2000, Dom Pedrito, RS. Anais... Dom Pedrito: Sindicato Rural de Dom Pedrito, 2000. v.8, p.1-27.

NABINGER, C. Manejo e utilização sustentável de pastagens. In: GOTTSCHAL, C.S. et al. (Orgs). CICLO DE PALESTRAS EM PRODUÇÃO E MANEJO DE BOVINOS DE CORTE, 3., 1998, Canoas, RS. Anais... Canoas: ULBRA, 1998. v.3, p.40 -105 .
PANDEY, C. B.; SINGH, J. S. Influence of grazing and soil conditions on secondary savanna vegetation in Índia. Journal of Vegetation Science, Uppsala, v.2, n.1, p.95-102, 1991.

PERIN, R. Desempenho de uma pastagem nativa melhorada sob pastejo contínuo e rotativo. 1990. $128 \mathrm{f}$. Dissertação (Mestrado em Zootecnia) - Programa de Pósgraduação em Agronomia, Universidade Federal do Rio Grande do Sul.

PILLAR, V.D.P. Multivariate exploratory analysis and randomization testing with MULTIV. Coenoses, Gorizia, v.12, n.2/3, p.145-148, 1997.

RIZO, L.M. et al. Desempenho de pastagem nativa e pastagem sobre-semeada com forrageiras hibernais com e sem glifosato. Ciência Rural, Santa Maria, v.34, n.6, p.1921-1926, 2004.

TOTHILL, J.C. et al. BOTANAL - A comprehensive sampling and computing procedure for estimating pasture yield and composition. 1. Field sampling. Tropical Agronomy Technical Memorandum, Camberra, v.78, n.7, p.1-24, 1992. 